\title{
The impact of fruit flavonoids on memory and cognition
}

\author{
Jeremy P. E. Spencer ${ }^{1,2 *}$ \\ ${ }^{1}$ Molecular Nutrition Group, School of Chemistry, Food and Pharmacy, University of Reading, Reading RG2 6AP, UK \\ ${ }^{2}$ Centre for Integrative Neuroscience and Neurodynamics, University of Reading, Reading RG2 6AP, UK
}

(Received 26 January 2010 - Revised 14 July 2010 - Accepted 20 July 2010)

There is intense interest in the studies related to the potential of phytochemical-rich foods to prevent age-related neurodegeneration and cognitive decline. Recent evidence has indicated that a group of plant-derived compounds known as flavonoids may exert particularly powerful actions on mammalian cognition and may reverse age-related declines in memory and learning. In particular, evidence suggests that foods rich in three specific flavonoid sub-groups, the flavanols, anthocyanins and/or flavanones, possess the greatest potential to act on the cognitive processes. This review will highlight the evidence for the actions of such flavonoids, found most commonly in fruits, such as apples, berries and citrus, on cognitive behaviour and the underlying cellular architecture. Although the precise mechanisms by which these flavonoids act within the brain remain unresolved, the present review focuses on their ability to protect vulnerable neurons and enhance the function of existing neuronal structures, two processes known to be influenced by flavonoids and also known to underpin neuro-cognitive function. Most notably, we discuss their selective interactions with protein kinase and lipid kinase signalling cascades (i.e. phosphoinositide-3 kinase/Akt and mitogen-activated protein kinase pathways), which regulate transcription factors and gene expression involved in both synaptic plasticity and cerebrovascular blood flow. Overall, the review attempts to provide an initial insight into the potential impact of regular flavonoid-rich fruit consumption on normal or abnormal deteriorations in cognitive performance.

Ageing is associated with many common chronic neurodegenerative diseases and the precise cause of the neuronal degeneration underlying these disease and, indeed, normal brain ageing remains unclear. It is thought that several cellular and molecular events are involved, including increases in oxidative stress, impaired mitochondria function, activation of neuronal apoptosis, the deposition of aggregated proteins and excitotoxicity. Thus far, the majority of existing drug treatments for the treatment of neurodegenerative disorders are unable to prevent the underlying degeneration of neurons and consequently there is a desire to develop alternative therapies capable of preventing the progressive loss of specific neuronal populations. Since the neuropathology of many neurodegenerative diseases has been linked to increases in brain oxidative stress, historically, strong efforts have been directed at exploring the antioxidant strategies to combat neuronal damage. Indeed, there has been intense interest in the neuroprotective effects of a group of plant secondary metabolites known as polyphenols, which are powerful antioxidants in vitro. A large number of dietary intervention studies in human subjects ${ }^{(1)}$ and animals ${ }^{(2)}$, in particular those using foods and beverages derived from Vitis vinifera (grape), Camellia sinensis (tea), Theobroma cacao (cocoa) and Vaccinium spp. (blueberry), have demonstrated beneficial effects on human vascular function and on improving memory and learning ${ }^{(2-13)}$. While such foods and beverages differ greatly in chemical composition, macronutrient and micronutrient content and energy load/serving, they have in common that they are among the major dietary sources of a group of phytochemicals called flavonoids (for review on source, structure and bioavailability, refer to Spencer et al. and Rice-Evans et al. ${ }^{(14,15)}$ ).

Evidence has begun to emerge that these low molecular weight, non-nutrient components may be responsible for the beneficial effects of flavonoid-rich foods in vivo, through their ability to directly or indirectly interact with the brain's innate architecture for memory ${ }^{(16,17)}$. Historically, the biological actions of flavonoids on the brain were attributed to their ability to exert antioxidant actions ${ }^{(15)}$, through their ability to scavenge reactive species or through their possible influences on intracellular redox status ${ }^{(18)}$. However, it is now clear that this classical hydrogen-donating antioxidant activity cannot account for the bioactivity of flavonoids in vivo, particularly in the brain, where they are found at only very low concentrations $^{(19)}$. Instead, it has been postulated that their effects in the brain are mediated by an ability to protect vulnerable neurons, enhance existing neuronal function, stimulate brain blood flow and induce neurogenesis ${ }^{(6)}$. In vitro work has indicated that flavonoids and their physiological metabolites are capable of inducing neuronal and glial signalling pathways crucial in inducing synaptic plasticity ${ }^{(20-22)}$, but only at low nanomolar concentrations ${ }^{(23)}$ similar to that reported in the brain $^{(16)}$. However, their interaction with these pathways has wider relevance, as these signalling pathways are also responsible for determining the fate of neurons following exposure to neurotoxins ${ }^{(24)}$ and inflammatory mediators ${ }^{(17)}$ and in controlling cerebrovascular blood flow. The present review examines 
the potential for flavonoids and flavonoid-rich fruits to influence brain function and the mechanisms that might be responsible for such actions in the brain.

\section{Modulation of memory and cognition by flavonoid-rich fruits}

A recent prospective study has provided strong evidence that dietary flavonoid intake is associated with better cognitive evolution, i.e. the preservation of cognitive performance with ageing ${ }^{(25)}$. Furthermore, there is much evidence to suggest that flavonoids found in fruits and fruit juices (most notably flavanols, flavanones and anthocyanins) have the capacity to improve memory ${ }^{(6,19,26-28)}$. A number of animal intervention studies, using diets containing between 1 and $2 \%(\mathrm{w} / \mathrm{w})$ freeze-dried fruit/fruit juice, have indicated that grape, pomegranate, strawberry and blueberry, as well as pure flavonoids (epicatechin and quercetin), are capable of affecting several aspects of memory and learning, notably rapid $^{(11)}$ and slow ${ }^{(29-32)}$ memory acquisition, short-term working memory ${ }^{(16,33-36)}$, long-term reference memory ${ }^{(9,37)}$, reversal learning ${ }^{(1,33)}$ and memory retention/retrieval ${ }^{(38)}$. For example, fruits such as strawberry, blueberry and blackberry (all rich in anthocyanins and flavanols) have been shown to be beneficial in retarding functional, age-related CNS and cognitive behavioural deficits ${ }^{(29,39,40)}$. There is also extensive evidence that berries, most notably blueberries, which are equally rich in both anthocyanin and flavanols, are effective at reversing age-related deficits in spatial working memory ${ }^{(16,35,37,40-46)}$. Furthermore, the effects of blueberry and blackberry appear to be most pronounced in terms of short-term memory, suggesting that these improvements are, in part, dependent on CA3-CA3 excitatory connections in the hippocampus ${ }^{(40,47)}$. Although it is presently uncertain as to whether it is the flavonoids within these fruits which are causal agents in inducing the behavioural effects, evidence is beginning to emerge that suggests they are able to induce both behavioural and related cellular changes. For example, the flavanol ( -$)$-epicatechin $(500 \mu \mathrm{g} / \mathrm{g})$, which is found in a variety of fruits (apple, pear, grape and all berries), has been shown to enhance the retention of rat spatial memory in water maze tasks, especially when combined with exercise ${ }^{(38)}$. This improvement was associated with increased angiogenesis and neuronal spine density in the dentate gyrus (DG) of the hippocampus and with the up-regulation of genes associated with learning in the hippocampus.

Alternatively, the blueberry-derived flavonoids may act to enhance the efficiency of spatial memory indirectly by acting on the DG, the hippocampal sub-region most sensitive to the effects of ageing ${ }^{(48)}$. DG granule cells are particularly vulnerable to the ageing process ${ }^{(49,50)}$, with age-dependent degeneration resulting in an impairment of information transfer between DG and CA3, thus resulting in an inability of CA3 networks to build new spatial representations ${ }^{(48)}$. This is supported by observations that DG lesioned animals exhibit marked difficulties in acquiring spatial representations ${ }^{(51)}$. Blueberry supplementation has been shown to significantly increase the proliferation of precursor cells in the DG of aged rats ${ }^{(37)}$. This link between DG neurogenesis, cognitive performance and ageing is well documented ${ }^{(52-56)}$ and may represent another mechanism by which fruits rich in flavonoids may improve memory by acting on the hippocampus. Again, it is unclear at present whether flavonoids themselves are wholly responsible for the effects of flavonoid-rich fruits in vivo and also whether they induce global changes in hippocampal (and other brain region) morphology/function or are they capable of more specific changes in hippocampal sub-regions. In the next section, we examine the known interactions of flavonoids with the cellular structures and processes involved in normal brain function in an attempt to better understand how these actions might underpin the wide range of beneficial actions of flavonoid-rich fruits on mammalian cognitive processing.

\section{Mechanisms of action}

There is now much evidence to suggest that fruit-derived phytochemicals, in particular flavonoids, are capable of promoting beneficial effects on memory and learning ${ }^{(16,26,29,39,43,57-59)}$. It appears that they are able to impact upon memory through their ability to exert effects directly on the brain's innate architecture for memory ${ }^{(6)}$. This cellular architecture is well known to deteriorate with ageing with neuronal populations or synaptic connections lost over time, leaving the system less efficient in the processing and storage of sensory information. The next three sections describe how specific flavonoids or flavonoid-rich fruits impact upon this innate cellular architecture and thereby influence cognitive processing and ultimately behavioural outcomes such as memory.

\section{Interaction with neuronal signalling and synaptic function}

The ability of flavonoids to impact upon memory appears to be, in part, underpinned by their ability to interact with the molecular and physiological apparatus used in normal memory processing ${ }^{(21,60)}$. The concentrations of flavonoids and their metabolites that reach the brain following dietary supplementation are believed to be in the region of $10-300 \mathrm{~nm}$. Such concentrations are sufficiently high to exert pharmacological activity at receptors, kinases and transcription factors ${ }^{(20)}$. Although the precise site of their interaction with signalling pathways remains unresolved, evidence indicates that they are capable of acting in a number of ways: (1) by binding to ATP sites on enzymes and receptors; (2) by modulating the activity of kinases directly, i.e. MAPKKK, MAPKK or MAPK; (3) by affecting the function of important phosphatases, which act in opposition to kinases; (4) by modulating transcription factor activation and binding to promoter sequences, i.e. cyclicAMP-response element-binding protein (reviewed in Spencer ${ }^{(21,61)}$ ) (Fig. 1).

Flavonoids and flavonoid-rich fruits are well reported to modulate neuronal signalling pathways crucial in inducing synaptic plasticity ${ }^{(21)}$, in particular with the extracellular receptor kinase and protein kinase B/Akt pathways ${ }^{(61-63)}$. The activation of these pathways has been observed in vivo following dietary intervention with blueberry $(2 \% \quad(w / w)$ freeze-dried blueberry), along with the activation of the transcription factor cyclicAMP-response element-binding protein and production of neurotrophins such as brain-derived neurotrophic factor, which are known to be required during memory acquisition and consolidation. Agents, both dietary and otherwise, capable of inducing pathways leading to 


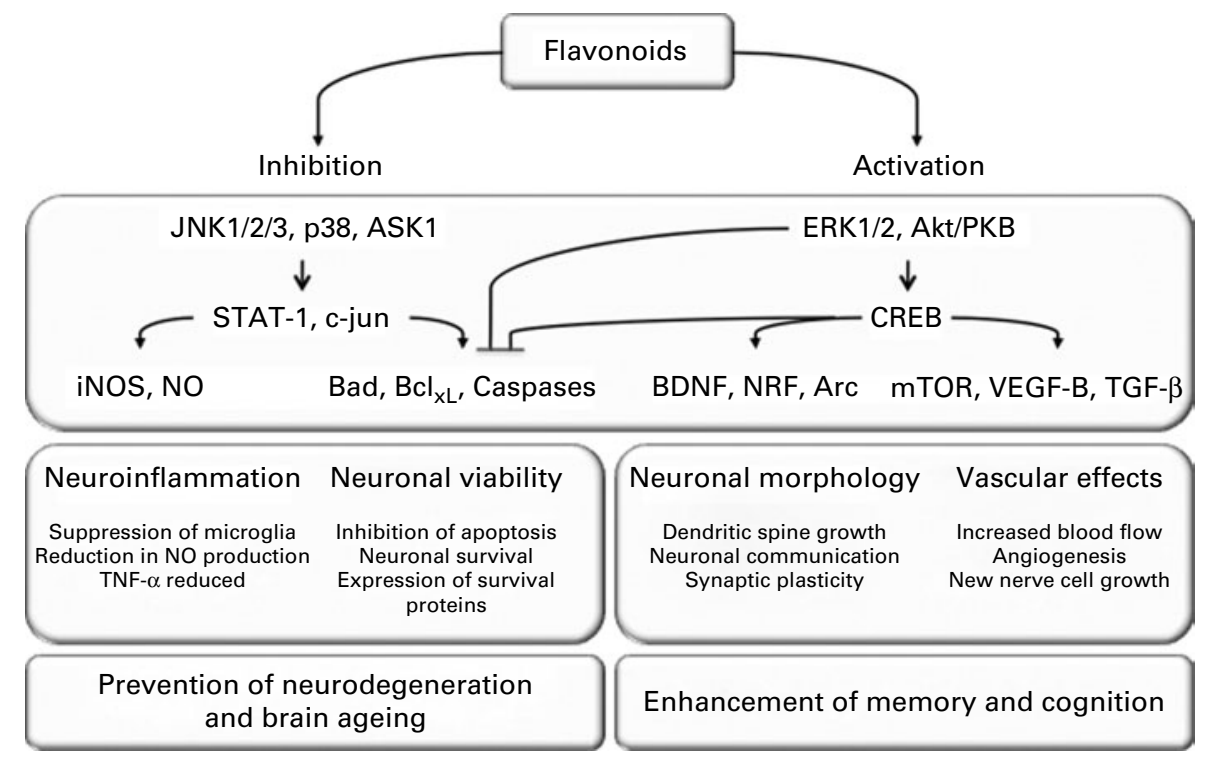

Fig. 1. Flavonoid-induced activation and inhibition of neuronal and glial signalling and functional implications. Activation of extracellular receptor kinase (ERK), Akt and cyclicAMP-response element-binding protein (CREB) by flavonoids may promote changes in synaptic plasticity and neurogenesis, which ultimately influence memory, learning and cognition. Activation of these pathways may also lead to the inhibition of pro-apoptotic signalling in neurons (bad and caspases). Flavonoid-induced inhibition of the c-jun N-terminal kinases (JNK), apoptosis signal-regulating kinase-1 and p38 pathways leads to an inhibition of both the apoptosis in neurons and a reduction of neuroinflammatory reactions in microglia (reduction in inductible nitric oxide synthase (iNOS) expression and NO- release). PKB, protein kinase B; mTOR, mammalian target of rapamycin; STAT-1, signal transducers and activators of transcription family-1; c-jun, c-jun N-terminal kinases; NO, nitric oxide; BDNF, brain-derived neurotrophic factor; VEGF, vascular endothelial growth factor; TGF, tumour growth factor.

cyclicAMP-response element-binding protein activation are believed to have the potential to enhance both short-term and long-term memories ${ }^{(16)}$, through the initiation of processes leading to the generation of a more efficient structure for interpreting afferent nerve or sensory information. One mechanism by which this may come about is through flavonoid-induced increases in neuronal spine density and morphology, two factors considered vital for learning and memory ${ }^{(64)}$.

Changes in spine density, morphology and motility have been shown to occur with paradigms that induce synaptic, as well as altered sensory experience, and lead to alterations in synaptic connectivity and strength between neuronal partners, which ultimately affects the efficacy of synaptic communication (Fig. 1). In support of this, dietary supplementation with blueberries rich in both high flavanol and anthocyanin has been shown to cause activation of mammalian target of rapamycin and an increased expression of hippocampal Arc/ Arg3.1 ${ }^{(16)}$, events which are likely to facilitate changes in synaptic strength through the stimulation of the growth of small dendritic spines into large mushroom-shaped spines. The ability of flavonoids to induce such morphological changes through interactions with neuronal signalling is supported by studies which have shown that specific flavanols are capable of inducing neuronal dendrite outgrowth ${ }^{(65)}$. Furthermore, nobiletin, a poly-methoxylated flavone found in citrus peel, also induces neurite outgrowth ${ }^{(66)}$ and synaptic transmission $^{(67)}$ via its ability to interact directly with mitogen-activated protein kinase and PKA signalling pathways, while its metabolite, 4'-demethylnobiletin, exerts similar effects via the same pathways ${ }^{(68)}$. While these effects are interesting, and in agreement with previous observations with flavonoids, it should be noted that they were observed at concentrations ranging from 10 to $100 \mu \mathrm{M}$, which are unlikely to be achieved in the brain.

\section{Influence on blood flow and neurogenesis}

There is also evidence to suggest that flavonoid-rich foods may be capable of preventing many forms of cerebrovascular disease including those associated with stroke and dementia ${ }^{(69,70)}$. It is thought that flavonoids meditate these effects in vivo through their potential to affect endothelial function and peripheral blood flow ${ }^{(71)}$. Such vascular effects are potentially significant as increased cerebrovascular function is known to facilitate adult neurogenesis in the hippocampus ${ }^{(72)}$ (Fig. 1). Indeed, new hippocampal cells are clustered near blood vessels, which proliferate in response to vascular growth factors and may influence memory ${ }^{(73)}$. Efficient cerebral blood flow (CBF) is vital for optimal brain function, with several studies indicating that there is a decrease in $\mathrm{CBF}$ in patients with dementia ${ }^{(74,75)}$. Brain imaging techniques, such as 'functional MRI' and 'trans-cranial Doppler ultrasound', have shown that there is a correlation between $\mathrm{CBF}$ and cognitive function in human subjects ${ }^{(75)}$. For example, $\mathrm{CBF}$ velocity is significantly lower in patients with Alzheimer disease and low CBF is also associated with incipient markers of dementia. In contrast, non-demented subjects with higher CBF were less likely to develop dementia.

In this context, flavanol-rich foods have been shown to cause significantly increased CBF in human subjects, $1-2 \mathrm{~h}$ postintervention $^{(76,77)}$. Although requiring further investigation, intervention with a flavanol-rich drink derived from cocoa (400-900 mg flavanols) resulted in an acute ( $2 \mathrm{~h}$ postintervention) increase in blood flow (blood oxygen level-dependent functionalMRI) in certain regions of the brain, along with a modification of the blood oxygen level-dependent response in individuals completing a 'task switching' test. Furthermore, 'arterial spin-labelling sequence MRI'(78) also indicated that cocoa flavanols increase $\mathrm{CBF}$ up to a maximum of $2 \mathrm{~h}$ after 
ingestion of the flavanol-rich drink. In support of these findings, an increase in $\mathrm{CBF}$ through the middle cerebral artery has been reported after the consumption of flavanol-rich cocoa using trans-cranial Doppler ultrasound ${ }^{(77)}$. Clearly, further investigation is required before one can be certain of the full impact of flavonoids-rich foods on brain blood flow. At present, there is very little information regarding the ability of other flavonoid-rich foods, including effect of fruits on cerebrovascular blood flow. However, if such responses are ultimately dependent on the actions of flavanols on the vascular system, as has been suggested ${ }^{(71)}$, then there is good reason to hypothesise that other flavanol-rich foods, such as apple, grape, blackcurrant and pear, may also possess similar activity.

\section{Inhibition of neurodegeneration and neuroinflammation}

The underlying neurodegeneration observed in Parkinson's, Alzheimer's and other neurodegenerative diseases is believed to be triggered by multi-factorial processes, including neuroinflammation $^{(79,80)}$, glutamatergic excitotoxicity and increases in $\mathrm{Fe}$ and/or depletion of endogenous antioxidants ${ }^{(81-83)}$. There is a growing body of evidence to suggest that flavonoids and flavonoid-rich foods may be capable of counteracting such neuronal injury, thereby delaying the progression of disease pathologies $^{(19,22,61,84)}$. The death of nigral neurons in Parkinson's disease is thought to involve the formation of the endogenous neurotoxin, 5-S-cysteinyl-dopamine and its oxidation product, dihydrobenzothiazine- $1^{(5,85-87)}$. However, the generation of 5 -S-cysteinyl-dopamine ${ }^{(88)}$ and resulting neuronal injury induced by it are effectively counteracted by a range of flavonoids and other polyphenols found commonly in a range of fruits such as orange, berries, apple and grape ${ }^{(5)}$. There is also evidence that flavanols and their metabolites are effective in blocking oxidant-induced neuronal injury ${ }^{(89,90)}$ at concentrations relevant to those observed in vivo and in the brain (typically $10-300 \mathrm{~nm}$ ), through their ability to modulate PI3 kinase (PI3K)/Akt and mitogen-activated protein kinase signalling ${ }^{(20,61)}$. For example, the flavanols epicatechin and $3^{\prime}$-O-methyl-epicatechin (100 and $\left.300 \mathrm{~nm}\right)$ protect neurons against oxidative damage via a mechanism involving the suppression of c-jun $N$-terminal kinases, and downstream partners, c-jun and pro-caspase- $3^{(91)}$ (Fig. 1). Similarly, the citrus flavanones, hesperetin and its metabolite, 5-nitro-hesperetin $(10-300 \mathrm{~nm})$, inhibit oxidant-induced neuronal apoptosis via a mechanism involving the activation/phosphorylation of signalling proteins important in the pro-survival pathways ${ }^{(92)}$.

Recent evidence suggests that non-steroidal, anti-inflammatory drugs are effective in delaying the onset of neurodegenerative disorders, particularly Parkinson's disease ${ }^{(93)}$. As such, there has been an interest in the development of new compounds with an ability to counteract neuroinflammatory injury to the brain. The citrus flavanone naringenin $(300 \mathrm{~nm})$ has recently been found to be highly effective in reducing lipopolysaccharide/interferon- $\gamma$-induced glial-cell activation and resulting neuronal injury, via inhibition of p38 and signal transducers and activators of transcription family-1, and a reduction in inductible nitric oxide synthase expression and other flavonoids has been shown to partially alleviate neuroinflammation through the inhibition of TNF- $\alpha$ production ${ }^{(94)}$ (Fig. 1). Flavonoids present in blueberry have also been shown to inhibit $\mathrm{NO} \cdot \mathrm{IL}-1 \beta$ and $\mathrm{TNF}-\alpha$ production in activated microglia cells ${ }^{(95)}$, while the flavonol quercetin $(1-30 \mu \mathrm{M})^{(96)}$ and the flavanols catechin and epigallocatechin gallate $(1-50 \mu \mathrm{M})^{(97)}$ have all been shown to attenuate microglia- and/or astrocyte-mediated neuroinflammation. As with their activity against oxidative stress, their ability to exert such actions appears to rely on their ability to directly modulate kinase signalling pathways, pro-inflammatory transcription factors and the downstream regulation of inductible nitric oxide synthase and cyclooxygenase-2 expression, NO production, cytokine release and NADPH oxidase activation $^{(20,21,61,98)}$. For example, flavonol fisetin $(1 \mu \mathrm{M})$, which is found in strawberry and other fruits, has been shown to inhibit p38 mitogen-activated protein kinase phosphorylation in LPS-stimulated BV-2 microglial cells ${ }^{(99)}$ and the flavone luteolin $(5-50 \mu \mathrm{M})$ inhibits IL-6 production in activated microglia via inhibition of the c-jun $N$-terminal kinases signalling pathway ${ }^{(100)}$.

\section{Summary and future horizons}

The actions of flavonoid-rich fruits and the specific flavonoids that they contain on brain function appear to express significant similarity. This suggests that the ability of many fruits to exert effects on cognition appears to be underpinned by their flavonoid content and involves a number of effects, including a potential to protect neurons against injury induced by neurotoxins and neuroinflammation, a potential to activate synaptic signalling and an ability to improve cerebrovascular blood flow. These effects appear to be mediated by the interaction of flavonoids and their physiological metabolites with cellular signalling cascades in the brain and the periphery, leading to an inhibition of apoptosis triggered by neurotoxic species, the promotion of neuronal survival and differentiation and an enhancement of peripheral and cerebral blood perfusion. Such effects induce beneficial changes in the cellular architecture required for cognition and consequently provide the brain with a more efficient structure for interpreting afferent nerve or sensory information and for the storage, processing and retrieval of memory. Furthermore, such interactions also protect the brain against neuronal losses associated with ageing, something which is particularly relevant as this innate brain structure is known to deteriorate with ageing, with neuronal populations or synaptic connections lost over time, leaving the system less efficient in its ability to process sensory information.

The consumption of flavonoid-rich fruits, such as berries, apple and citrus, throughout life may have the potential to limit or even reverse age-dependent deteriorations in memory and cognition. However, there are a number of questions still to be resolved. Most notably, at present, there is no data in support of a causal relationship between the consumption of flavonoids and behavioural outcomes in human subjects. In order to make such relationships, future intervention studies will be required to utilise better-characterised intervention materials, more appropriate controls and more rigorous clinical outcomes. While cognitive behavioural testing in human subjects and animals provides an appropriate way of assessing function, in vivo structural and dynamic quantitative assessments will ultimately be required to provide hard evidence of effects in the brain. For example, it would be 
highly advantageous to directly link behavioural responses to changes in hippocampal volume and density, changes in neural stem cell and progenitor cells and alterations in brain blood flow using MRI and functional MRI techniques. Functional MRI measures may be used to assess changes in blood flow that underlie improved cognitive functioning as a result of flavonoid-rich fruit supplementation. In addition, such haemodynamic changes may be further compared with changes in grey matter density and with biomarkers of neural stem and progenitor cells, using proton NMR spectroscopy. Such an approach will be essential to provide links between flavonoid intake and brain function in a mechanistic, dynamic and quantitative way. Taking such an approach, one may also be able to assess other factors relating to intake such as the timeframe required to gain maximum beneficial effects, the flavonoids most effective in inducing these changes and the doses at which these become effective?

Furthermore, it appears that the precise mechanism by which flavonoids act on cognitive performance appears to be dependent on the period of flavonoid exposure. At present, improvements in cognition resulting from acute dietary flavonoid-rich fruit interventions are thought to be dependent on increased cerebrovascular blood flow. However, in vitro studies using physiological doses of flavonoids have shown that they are able to rapidly stimulate neuronal signalling pathways involved in cognitive processing and thus even acute changes in cognition may be partly mediated by their direct actions on neurons. In order to resolve this issue, further studies are necessary to clearly resolve the issue of whether flavonoids are able to localise in the brain following the consumption of flavonoid-rich fruits. In human subjects, this can only be resolved by the use of brain imaging technologies allied to intervention studies utilising ${ }^{13} \mathrm{C}$-labelled flavonoids (either pure ${ }^{13} \mathrm{C}$-labelled flavonoids or with fruits harvested from plants grown in a ${ }^{13} \mathrm{CO}_{2}$ environment). Cognitive changes associated with long-term intake of fruit flavonoids are more likely to involve morphological changes triggered by the direct actions of flavonoids on neuronal signalling. However, the extent to which daily acute changes in brain blood flow impact upon such changes are presently unknown. Thus, future studies are necessary and should be designed to resolve the precise temporal nature of their effects on memory as well as other issues, such as when one needs to start consuming flavonoids to gain the maximum beneficial effects?

Finally, the potential impact of diet on health care costs should not be ignored. Dementia costs to the UK alone have been estimated to be $£ 17$ billion/annum. If scientists could develop a treatment that would reduce severe cognitive impairment in older people by just $1 \%$ per year, this would cancel out all estimated increases in the long-term care costs due to our ageing population (Alzheimer's Research Trust). Beyond this, there is also intense interest in the development of drugs capable of enhancing memory and learning, both in adults and in children, and there is a strong possibility that in the future, specific nutrients, in particular fruit-derived flavonoids, might act as precursors for the development of a new generation of memory-enhancing drugs. As such, the present series of reviews in this issue are extremely timely one and highlight the present thinking in the field and outline the future directions for research in the area.

\section{Acknowledgements}

J. P. E. S. is funded by the Biotechnology and Biological Sciences Research Council (BB/F008953/1; BB/E023185/1; $\mathrm{BB} / \mathrm{G} 005702 / 1$ ), the FSA (FLAVURS) and the European Union (FP7 FLAVIOLA). There is no conflict of interest that I should disclose, having read the Journals guidelines. J. P. E. S. is the sole author of the manuscript.

\section{References}

1. Macready AL, Kennedy OB, Ellia JA, et al. (2009) Flavonoids and cognitive function: a review of human randomized controlled trial studies and recommendations for future studies. Genes Nutr 4, 227-242.

2. Rendeiro C, Spencer JPE, Vauzour D, et al. (2009) The impact of flavonoids on spatial memory in rodents: from behaviour to underlying hippocampal mechanisms. Genes Nutr 4, 251-270.

3. Spencer JPE, Vauzour D \& Rendeiro C (2009) Flavonoids and cognition: the molecular mechanisms underlying their behavioural effects. Arch Biochem Biophys 492, 1-9.

4. Checkoway H, Powers K, Smith-Weller T, et al. (2002) Parkinson's disease risks associated with cigarette smoking, alcohol consumption, and caffeine intake. Am J Epidemiol 155, 732-738.

5. Vauzour D, Ravaioli G, VafeiAdou K, et al. (2008) Peroxynitrite induced formation of the neurotoxins 5-Scysteinyl-dopamine and DHBT-1: implications for Parkinson's disease and protection by polyphenols. Arch Biochem Biophys 476, 145-151.

6. Spencer JPE (2008) Food for thought: the role of dietary flavonoids in enhancing human memory, learning and neurocognitive performance. Proc Nutr Soc 67, 238-252.

7. Galli RL, Shukitt-Hale B, Youdim KA, et al. (2002) Fruit polyphenolics and brain aging: nutritional interventions targeting age-related neuronal and behavioral deficits. Ann N Y Acad Sci 959, 128-132.

8. Unno K, Takabayashi F, Kishido T, et al. (2004) Suppressive effect of green tea catechins on morphologic and functional regression of the brain in aged mice with accelerated senescence (SAMP10). Exp Gerontol 39, 1027-1034.

9. Haque AM, Hashimoto M, Katakura M, et al. (2006) Long-term administration of green tea catechins improves spatial cognition learning ability in rats. J Nutr 136, 1043-1047.

10. Kuriyama S, Hozawa A, Ohmori K, et al. (2006) Green tea consumption and cognitive function: a cross-sectional study from the Tsurugaya Project 1. Am J Clin Nutr 83, 355-361.

11. Wang Y, Wang L, Wu J, et al. (2006) The in vivo synaptic plasticity mechanism of EGb 761-induced enhancement of spatial learning and memory in aged rats. $\mathrm{Br} J$ Pharmacol 148, 147-153.

12. Youdim KA, Shukitt-Hale B \& Joseph JA (2004) Flavonoids and the brain: interactions at the blood-brain barrier and their physiological effects on the central nervous system. Free Radic Biol Med 37, 1683-1693.

13. Joseph J, Cole G, Head E, et al. (2009) Nutrition, brain aging, and neurodegeneration. J Neurosci 29, 12795-12801.

14. Spencer JPE, Abd El Mohsen MM, Minihane AM, et al. (2007) Biomarkers of the intake of dietary polyphenols: strengths, limitations and application in nutrition research. Br J Nutr 99, 12-22.

15. Rice-Evans CA, Miller NJ \& Paganga G (1996) Structureantioxidant activity relationships of flavonoids and phenolic acids. Free Radic Biol Med 20, 933-956.

16. Williams CM, El Mohsen MA, Vauzour D, et al. (2008) Blueberry-induced changes in spatial working memory 
correlate with changes in hippocampal CREB phosphorylation and brain-derived neurotrophic factor (BDNF) levels. Free Radic Biol Med 45, 295-305.

17. Spencer JPE (2009) Flavonoids and brain health: multiple effects underpinned by common mechanisms. Genes Nutr $\mathbf{4}$, 243-250.

18. Pollard SE, Kuhnle GG, Vauzour D, et al. (2006) The reaction of flavonoid metabolites with peroxynitrite. Biochem Biophys Res Commun 350, 960-968.

19. Spencer JPE (2008) Flavonoids: modulators of brain function? Br J Nutr 99E, Suppl. 1, ES60-ES77.

20. Williams RJ, Spencer JPE \& Rice-Evans C (2004) Flavonoids: antioxidants or signalling molecules? Free Radic Biol Med 36, $838-849$.

21. Spencer JPE (2009) The impact of flavonoids on memory: physiological and molecular considerations. Chem Soc Rev 38, $1152-1161$.

22. Vauzour D, VafeiAdou K, Rodriguez-Mateos A, et al. (2008) The neuroprotective potential of flavonoids: a multiplicity of effects. Genes Nutr 3, 115-126.

23. Vauzour D, VafeiAdou K, Rice-Evans C, et al. (2007) Activation of pro-survival Akt and ERK1/2 signalling pathways underlie the anti-apoptotic effects of flavanones in cortical neurons. J Neurochem 103, 1355-1367.

24. Weinreb O, Amit T, Mandel S, et al. (2009) Neuroprotective molecular mechanisms of (-)-epigallocatechin-3-gallate: a reflective outcome of its antioxidant, iron chelating and neuritogenic properties. Genes Nutr 4, 283-296.

25. Letenneur L, Proust-Lima C, Le GA, et al. (2007) Flavonoid intake and cognitive decline over a 10 -year period. Am J Epidemiol 165, 1364-1371.

26. Shukitt-Hale B, Lau FC \& Joseph JA (2008) Berry fruit supplementation and the aging brain. J Agric Food Chem 56, 636-641.

27. Shukitt-Hale B, Carey AN, Jenkins D, et al. (2007) Beneficial effects of fruit extracts on neuronal function and behavior in a rodent model of accelerated aging. Neurobiol Aging 28, 1187-1194.

28. Shukitt-Hale B, Lau FC \& Joseph JA (2008) Berry fruit supplementation and the aging brain. J Agric Food Chem 56, 636-641.

29. Joseph JA, Shukitt-Hale B, Denisova NA, et al. (1999) Reversals of age-related declines in neuronal signal transduction, cognitive, and motor behavioral deficits with blueberry, spinach, or strawberry dietary supplementation. J Neurosci 19, 8114-8121.

30. Shif O, Gillette K, Damkaoutis CM, et al. (2006) Effects of Ginkgo biloba administered after spatial learning on water maze and radial arm maze performance in young adult rats. Pharmacol Biochem Behav 84, 17-25.

31. Hartman RE, Shah A, Fagan AM, et al. (2006) Pomegranate juice decreases amyloid load and improves behavior in a mouse model of Alzheimer's disease. Neurobiol Dis 24, 506-515.

32. Winter JC (1998) The effects of an extract of Ginkgo biloba, EGb 761, on cognitive behavior and longevity in the rat. Physiol Behav 63, 425-433.

33. Hoffman JR, Donato A \& Robbins SJ (2004) Ginkgo biloba promotes short-term retention of spatial memory in rats. Pharmacol Biochem Behav 77, 533-539.

34. Walesiuk A, Trofimiuk E \& Braszko JJ (2006) Ginkgo biloba normalizes stress- and corticosterone-induced impairment of recall in rats. Pharmacol Res 53, 123-128.

35. Ramirez MR, Izquierdo I, do Carmo Bassols RM, et al. (2005) Effect of lyophilised Vaccinium berries on memory, anxiety and locomotion in adult rats. Pharmacol Res 52, 457-462.

36. Pu F, Mishima K, Irie K, et al. (2007) Neuroprotective effects of quercetin and rutin on spatial memory impairment in an 8-arm radial maze task and neuronal death induced by repeated cerebral ischemia in rats. J Pharmacol Sci 104, 329-334.

37. Casadesus G, Shukitt-Hale B, Stellwagen HM, et al. (2004) Modulation of hippocampal plasticity and cognitive behavior by short-term blueberry supplementation in aged rats. Nutr Neurosci 7, 309-316.

38. van Praag H, Lucero MJ, Yeo GW, et al. (2007) Plant-derived flavanol (-)-epicatechin enhances angiogenesis and retention of spatial memory in mice. J Neurosci 27, 5869-5878.

39. Joseph JA, Shukitt-Hale B, Denisova NA, et al. (1998) Longterm dietary strawberry, spinach, or vitamin E supplementation retards the onset of age-related neuronal signal-transduction and cognitive behavioral deficits. J Neurosci 18, 8047-8055.

40. Shukitt-Hale B, Cheng V \& Joseph JA (2009) Effects of blackberries on motor and cognitive function in aged rats. Nutr Neurosci 12, 135-140.

41. Andres-Lacueva C, Shukitt-Hale B, Galli RL, et al. (2005) Anthocyanins in aged blueberry-fed rats are found centrally and may enhance memory. Nutr Neurosci 8, 111-120.

42. Goyarzu P, Malin DH, Lau FC, et al. (2004) Blueberry supplemented diet: effects on object recognition memory and nuclear factor-kappa B levels in aged rats. Nutr Neurosci 7, $75-83$.

43. Joseph JA, Denisova NA, Arendash G, et al. (2003) Blueberry supplementation enhances signaling and prevents behavioral deficits in an Alzheimer disease model. Nutr Neurosci 6, $153-162$.

44. Barros D, Amaral OB, Izquierdo I, et al. (2006) Behavioral and genoprotective effects of Vaccinium berries intake in mice. Pharmacol Biochem Behav 84, 229-234.

45. Willis LM, Shukitt-Hale B \& Joseph JA (2009) Recent advances in berry supplementation and age-related cognitive decline. Curr Opin Clin Nutr Metab Care 12, 91-94.

46. Barros D, Amaral OB, Izquierdo I, et al. (2006) Behavioral and genoprotective effects of Vaccinium berries intake in mice. Pharmacol Biochem Behav 84, 229-234.

47. Rolls ET \& Kesner RP (2006) A computational theory of hippocampal function, and empirical tests of the theory. Prog Neurobiol 79, 1-48.

48. Burke SN \& Barnes CA (2006) Neural plasticity in the ageing brain. Nat Rev Neurosci 7, 30-40.

49. Small SA, Tsai WY, DeLaPaz R, et al. (2002) Imaging hippocampal function across the human life span: is memory decline normal or not? Ann Neurol 51, 290-295.

50. Small SA, Chawla MK, Buonocore M, et al. (2004) Imaging correlates of brain function in monkeys and rats isolates a hippocampal subregion differentially vulnerable to aging. Proc Natl Acad Sci U S A 101, 7181-7186.

51. Xavier GF, Oliveira-Filho FJ \& Santos AM (1999) Dentate gyrus-selective colchicine lesion and disruption of performance in spatial tasks: difficulties in 'place strategy' because of a lack of flexibility in the use of environmental cues? Hippocampus 9, 668-681.

52. Kuhn HG, Dickinson-Anson H \& Gage FH (1996) Neurogenesis in the dentate gyrus of the adult rat: age-related decrease of neuronal progenitor proliferation. J Neurosci 16, 2027-2033.

53. Kempermann G, Kuhn HG \& Gage FH (1998) Experienceinduced neurogenesis in the senescent dentate gyrus. J Neurosci 18, 3206-3212.

54. Drapeau E, Mayo W, Aurousseau C, et al. (2003) Spatial memory performances of aged rats in the water maze predict levels of hippocampal neurogenesis. Proc Natl Acad Sci U S A 100, 14385-14390.

55. Shors TJ, Townsend DA, Zhao M, et al. (2002) Neurogenesis may relate to some but not all types of hippocampal-dependent learning. Hippocampus 12, 578-584. 
56. Stangl D \& Thuret S (2009) Impact of diet on adult hippocampal neurogenesis. Genes Nutr 4, 271-282.

57. Joseph JA, Shukitt-Hale B \& Casadesus G (2005) Reversing the deleterious effects of aging on neuronal communication and behavior: beneficial properties of fruit polyphenolic compounds. Am J Clin Nutr 81, 313S-316S.

58. Joseph JA, Shukitt-Hale B \& Lau FC (2007) Fruit polyphenols and their effects on neuronal signaling and behavior in senescence. Ann N Y Acad Sci 1100, 470-485.

59. Shukitt-Hale B, Carey A, Simon L, et al. (2006) Effects of Concord grape juice on cognitive and motor deficits in aging. Nutrition 22, 295-302.

60. Joseph JA, Shukitt-Hale B \& Lau FC (2007) Fruit polyphenols and their effects on neuronal signaling and behavior in senescence. Ann N Y Acad Sci 1100, 470-485.

61. Spencer JPE (2007) The interactions of flavonoids within neuronal signalling pathways. Genes Nutr 2, 257-273.

62. Spencer JPE, Rice-Evans C \& Williams RJ (2003) Modulation of pro-survival Akt/protein kinase B and ERK1/2 signaling cascades by quercetin and its in vivo metabolites underlie their action on neuronal viability. $J$ Biol Chem 278, 34783-34793.

63. Schroeter H, Bahia P, Spencer JPE, et al. (2007) (-)-Epicatechin stimulates ERK-dependent cyclic AMP response element activity and upregulates GLUR2 in cortical neurons. J Neurochem 101, 1596-1606.

64. Harris KM \& Kater SB (1994) Dendritic spines: cellular specializations imparting both stability and flexibility to synaptic function. Annu Rev Neurosci 17, 341-371.

65. Reznichenko L, Amit T, Youdim MB, et al. (2005) Green tea polyphenol (-)-epigallocatechin-3-gallate induces neurorescue of long-term serum-deprived PC12 cells and promotes neurite outgrowth. $J$ Neurochem 93, 1157-1167.

66. Nagase H, Yamakuni T, Matsuzaki K, et al. (2005) Mechanism of neurotrophic action of nobiletin in PC12D cells. Biochemistry 44, 13683-13691.

67. Matsuzaki K, Miyazaki K, Sakai S, et al. (2008) Nobiletin, a citrus flavonoid with neurotrophic action, augments protein kinase A-mediated phosphorylation of the AMPA receptor subunit, GluR1, and the postsynaptic receptor response to glutamate in murine hippocampus. Eur $J$ Pharmacol 578, 194-200.

68. Al RM, Nakajima A, Saigusa D, et al. (2009) 4'-Demethylnobiletin, a bioactive metabolite of nobiletin enhancing PKA/ ERK/CREB signaling, rescues learning impairment associated with NMDA receptor antagonism via stimulation of the ERK cascade. Biochemistry 48, 7713-7721.

69. Commenges D, Scotet V, Renaud S, et al. (2000) Intake of flavonoids and risk of dementia. Eur J Epidemiol 16, 357-363.

70. Dai Q, Borenstein AR, Wu Y, et al. (2006) Fruit and vegetable juices and Alzheimer's disease: the Kame Project. Am J Med 119, 751-759.

71. Schroeter H, Heiss C, Balzer J, et al. (2006) (-)-Epicatechin mediates beneficial effects of flavanol-rich cocoa on vascular function in humans. Proc Natl Acad Sci U S A 103, $1024-1029$.

72. Gage FH (2000) Mammalian neural stem cells. Science 287, $1433-1438$.

73. Palmer TD, Willhoite AR \& Gage FH (2000) Vascular niche for adult hippocampal neurogenesis. J Comp Neurol $\mathbf{4 2 5}$, 479-494.

74. Nagahama Y, Nabatame H, Okina T, et al. (2003) Cerebral correlates of the progression rate of the cognitive decline in probable Alzheimer's disease. Eur Neurol 50, 1-9.

75. Ruitenberg A, den Heijer T, Bakker SL, et al. (2005) Cerebral hypoperfusion and clinical onset of dementia: the Rotterdam Study. Ann Neurol 57, 789-794.
76. Francis ST, Head K, Morris PG, et al. (2006) The effect of flavanol-rich cocoa on the fMRI response to a cognitive task in healthy young people. J Cardiovasc Pharmacol 47, Suppl. 2, S215-S220.

77. Fisher ND, Sorond FA \& Hollenberg NK (2006) Cocoa flavanols and brain perfusion. J Cardiovasc Pharmacol 47, Suppl. 2, S210-S214.

78. Wang Z, Fernandez-Seara M, Alsop DC, et al. (2008) Assessment of functional development in normal infant brain using arterial spin labeled perfusion MRI. Neuroimage 39, 973-978.

79. Hirsch EC, Hunot S \& Hartmann A (2005) Neuroinflammatory processes in Parkinson's disease. Parkinsonism Relat Disord 11, Suppl.1, S9-S15.

80. McGeer EG \& McGeer PL (2003) Inflammatory processes in Alzheimer's disease. Prog Neuropsychopharmacol Biol Psychiatry 27, 741-749.

81. Barzilai A \& Melamed E (2003) Molecular mechanisms of selective dopaminergic neuronal death in Parkinson's disease. Trends Mol Med 9, 126-132.

82. Jellinger KA (2001) Cell death mechanisms in neurodegeneration. J Cell Mol Med 5, 1-17.

83. Spires TL \& Hannan AJ (2005) Nature, nurture and neurology: gene-environment interactions in neurodegenerative disease. FEBS Anniversary Prize Lecture delivered on 27 June 2004 at the 29th FEBS Congress in Warsaw. FEBS $J$ 272, 2347-2361.

84. Mandel S \& Youdim MB (2004) Catechin polyphenols: neurodegeneration and neuroprotection in neurodegenerative diseases. Free Radic Biol Med 37, 304-317.

85. Spencer JPE, Whiteman M, Jenner P, et al. (2002) 5-SCysteinyl-conjugates of catecholamines induce cell damage, extensive DNA base modification and increases in caspase-3 activity in neurons. $J$ Neurochem 81, 122-129.

86. Hastings TG (1995) Enzymatic oxidation of dopamine: the role of prostaglandin H synthase. J Neurochem 64, 919-924.

87. Spencer JPE, Jenner P, Daniel SE, et al. (1998) Conjugates of catecholamines with cysteine and GSH in Parkinson's disease: possible mechanisms of formation involving reactive oxygen species. J Neurochem 71, 2112-2122.

88. Vauzour D, Vafeiadou K \& Spencer JP (2007) Inhibition of the formation of the neurotoxin 5-S-cysteinyl-dopamine by polyphenols. Biochem Biophys Res Commun 362, 340-346.

89. Spencer JPE, Schroeter H, Kuhnle G, et al. (2001) Epicatechin and its in vivo metabolite, 3'-O-methyl epicatechin, protect human fibroblasts from oxidative-stress-induced cell death involving caspase-3 activation. Biochem J 354, 493-500.

90. Spencer JPE, Schroeter H, Crossthwaithe AJ, et al. (2001) Contrasting influences of glucuronidation and $O$-methylation of epicatechin on hydrogen peroxide-induced cell death in neurons and fibroblasts. Free Radic Biol Med 31, 1139-1146.

91. Schroeter H, Spencer JPE, Rice-Evans C, et al. (2001) Flavonoids protect neurons from oxidized low-density-lipoproteininduced apoptosis involving c-Jun N-terminal kinase (JNK), c-Jun and caspase-3. Biochem J 358, 547-557.

92. Vauzour D, VafeiAdou K, Rice-Evans C, et al. (2007) Activation of pro-survival Akt and ERK1/2 signaling pathways underlie the anti-apoptotic effects of flavanones in cortical neurons. J Neurochem 103, 1355-1367.

93. Casper D, Yaparpalvi U, Rempel N, et al. (2000) Ibuprofen protects dopaminergic neurons against glutamate toxicity in vitro. Neurosci Lett 289, 201-204.

94. VafeiAdou K, Vauzour D, Lee HY, et al. (2009) The citrus flavanone naringenin inhibits inflammatory signalling in glial cells and protects against neuroinflammatory injury. Arch Biochem Biophys 484, 100-109.

95. Lau FC, Bielinski DF \& Joseph JA (2007) Inhibitory effects of blueberry extract on the production of inflammatory mediators 
in lipopolysaccharide-activated BV2 microglia. J Neurosci Res 85, 1010-1017.

96. Chen JC, Ho FM, Pei-Dawn LC, et al. (2005) Inhibition of iNOS gene expression by quercetin is mediated by the inhibition of IkappaB kinase, nuclear factor-kappa B and STAT1, and depends on heme oxygenase-1 induction in mouse BV-2 microglia. Eur J Pharmacol 521, 9-20.

97. Li R, Huang YG, Fang D, et al. (2004) (-)-Epigallocatechin gallate inhibits lipopolysaccharide-induced microglial activation and protects against inflammation-mediated dopaminergic neuronal injury. J Neurosci Res 78, 723-731.
98. Bhat NR, Zhang P, Lee JC, et al. (1998) Extracellular signal-regulated kinase and $\mathrm{p} 38$ subgroups of mitogen-activated protein kinases regulate inducible nitric oxide synthase and tumor necrosis factor-alpha gene expression in endotoxinstimulated primary glial cultures. J Neurosci 18, 1633-1641.

99. Zheng LT, Ock J, Kwon BM, et al. (2008) Suppressive effects of flavonoid fisetin on lipopolysaccharide-induced microglial activation and neurotoxicity. Int Immunopharmacol 8, 484-494.

100. Jang S, Kelley KW \& Johnson RW (2008) Luteolin reduces IL-6 production in microglia by inhibiting JNK phosphorylation and activation of AP-1. Proc Natl Acad Sci U S A 105, 7534-7539. 